\title{
Phrenic to musculocutaneous nerve transfer for traumatic brachial plexus injuries: analyzing respiratory effects on elbow flexion control
}

\author{
Mariano Socolovsky, MD, PhD,, Martijn Malessy, MD, PhD, ${ }^{1}$ Gonzalo Bonilla, MD, \\ Gilda Di Masi, MD, ${ }^{1}$ María Eugenia Conti, MD, ${ }^{3}$ and Ana Lovaglio, MD ${ }^{1}$ \\ ${ }^{1}$ Peripheral Nerve and Brachial Plexus Surgery Program, Department of Neurosurgery, and ${ }^{3}$ Unit of Neurophysiology, \\ Department of Neurology, University of Buenos Aires School of Medicine, Buenos Aires, Argentina; and 2Department of \\ Neurosurgery, University of Leiden School of Medicine, Leiden, Holland
}

\begin{abstract}
OBJECTIVE In this study, the authors sought to identify the relationship between breathing and elbow flexion in patients with a traumatic brachial plexus injury (TBPI) who undergo a phrenic nerve (PN) transfer to restore biceps flexion. More specifically, the authors studied whether biceps strength and the maximal range of active elbow flexion differ between full inspiration and expiration, and whether electromyography (EMG) activity in the biceps differs between forced maximum breathing during muscular rest, normal breathing during rest, and at maximal biceps contraction. All these variables were studied in a cohort with different intervals of follow-up, as the authors sought to determine if the relationship between breathing movements and elbow flexion changes over time.
\end{abstract}

METHODS The British Medical Research Council muscle-strength grading system and a dynamometer were used to measure biceps strength, which was measured 1) during a maximal inspiratory effort, 2) during respiratory repose, and 3) after a maximal expiratory effort. The maximum range of elbow flexion was measured 1) after maximal inspiration, 2) during normal breathing, and 3) after maximal expiration. Postoperative EMG testing was performed 1) during normal breathing with the arm at rest, 2) during sustained maximal inspiration with the arm at rest, and 3) during maximal voluntary biceps contraction. Within-group (paired) comparisons, and both correlation and regression analyses were performed.

RESULTS Twenty-one patients fit the study inclusion criteria. The mean interval from trauma to surgery was 5.5 months, and the mean duration of follow-up 2.6 years (range 10 months to 9.6 years). Mean biceps strength was 0.21 after maximal expiration versus 0.29 after maximal inspiration, a difference of $0.08(t=4.97, p<0.001)$. Similarly, there was almost a $21^{\circ}$ difference in maximum elbow flexion, from $88.8^{\circ}$ after expiration to $109.5^{\circ}$ during maximal inspiration $(t=5.05, p<0.001$ ). Involuntary elbow flexion movement during breathing was present in $18 / 21$ patients $(86 \%)$ and averaged almost $20^{\circ}$. Measuring involuntary EMG activity in the biceps during rest and contraction, there were statistically significant direct correlations between readings taken during normal and deep breathing, which were moderate $(r=0.66$, $p<0.001)$ and extremely strong $(r=0.94, p<0.001)$, respectively. Involuntary activity also differed significantly between normal and deep breathing $(2.14 \mathrm{vs} 3.14, t=4.58, p<0.001)$. The degrees of involuntary flexion were significantly greater within the first 2.6 years of follow-up than later.

CONCLUSIONS These results suggest that the impact of breathing on elbow function is considerable after PN transfer for elbow function reconstruction following a TBPI, both clinically and electromyographically, but also that there may be some waning of this influence over time, perhaps secondary to brain plasticity. In the study cohort, this waning impacted elbow range of motion more than biceps muscle strength and EMG recordings.

https://thejns.org/doi/abs/10.3171/2018.4.JNS173248

KEYWORDS brachial plexus injury; phrenic nerve transfer; brain plasticity; peripheral nerve 
B RAIN plasticity has been linked to the restored control of movements after nerve transfer surgery, performed to regain function in patients with brachial plexus injury (BPI). In those individuals in whom intercostal nerves (ICNs) are transferred to the musculocutaneous nerve $(\mathrm{MCN})$, the first sign of biceps recovery is the presence of involuntary contractions linked to breathing (the so-called "breathing arm"). Some months after surgery, patients come to realize that they can contract their biceps, but only through active inspiration or expiration. Eventually, contraction (and the maintenance of elbow flexion) becomes independent of respiration. Flexion can then be maintained while breathing normally. However, complete independence between the two functions is never actually achieved. ${ }^{1,4,7}$ This sequence of changes in the motor control pattern is likely mediated by brain plasticity, probably via the activation of a preexisting interneuronal network with normally low active connections between the two involved cortical areas. ${ }^{9}$

Phrenic nerve (PN) transfer for recovery of elbow flexion has been associated with a high rate of success. The overall effectiveness that our surgical group has achieved with this nerve transfer technique was published previously. Briefly, more than $60 \%$ of our patients achieve Grade 4 strength-using the British Medical Research Council (BMRC) muscle scale grading system-while more than $20 \%$ experience Grade 3 recovery. In summary, roughly $85 \%$ of our patients with a severe brachial palsy plexus experience good elbow flexion recovery. ${ }^{8}$ These results are consistent with others published in the literature. ${ }^{11}$

The main objective of the present study was to identify the relationship between breathing movements and elbow flexion in patients with a traumatic BPI (TBPI) who underwent a PN transfer to restore biceps flexion. More specifically, we studied whether elbow flexion strength and the maximal angle of elbow flexion differ between full inspiration and expiration, and whether electromyography (EMG) activity in the biceps differs between forced maximum breathing during muscular rest, normal breathing at muscular rest, and breathing at the point of maximal biceps contraction. All these variables were studied in a cohort of patients with different intervals of follow-up as we sought to determine if the relationship between breathing movements and elbow flexion changed over time.

\section{Methods \\ Inclusion Criteria}

We retrospectively reviewed patients with severe brachial plexus palsy for which a PN to musculocutaneous nerve $(\mathrm{MCN})$ transfer was performed to restore biceps muscle function. This type of nerve transfer is currently only used at our institution when no alternative intraplexal donors are available. All patients were operated upon consecutively in the Peripheral Nerve Unit within the Department of Neurosurgery, University of Buenos Aires School of Medicine, between January 1, 2005, and December 31,2016. To be included in the study, patients had to have suffered a severe injury (C5-T1 or C5-8) to exclude the possibility that preserved middle trunk elements might spontaneously reinnervate elbow flexion. Exclusion criteria were patients lost to follow-up, refusal to undergo an EMG study, surgical repair more than 12 months after the TBPI, an elbow fracture, an upper-limb amputation, or a postoperative outcome for the PN-MCN transfer of biceps muscle strength $<$ BMRC Grade 4 using the BMRC muscle-strength grading system. To be considered for this study, preoperatively all patients also had to have a normally functioning PN, determined by chest radiography and respiratory function tests, and intraoperatively by direct nerve stimulation. Preoperative evaluation of the brachial plexus lesion included neurophysiological studies (nerve conduction and EMG) and myelo-MRI to detect the presence of root avulsions.

The study was performed in full accordance with the Declaration of Helsinki II and our institution's ethics committee. All eligible patients were asked to participate in our study protocol, which included both clinical and electrophysiological testing. Patient selection for PN transfer was made based upon certain criteria: age from 16 to 35 years, normal respiratory function, no radiological abnormalities on chest radiography, no obvious ICN damage, and no severe thoracic cage trauma. Written informed consent was obtained from each patient prior to study participation. The minimum follow-up was 10 months for all patients included in the current analysis. Patient characteristics such as sex, age, time from trauma to surgery, and duration of follow-up were recorded at the time of assessment.

\section{Surgical Strategies and Techniques}

An incision was made one finger-breadth superior to the clavicle, which allowed for complete exposure of the proximal brachial plexus and its branches (including the upper and middle trunks with their anterior and posterior divisions, and the suprascapular nerve), the PN, and the spinal accessory nerve. Once available roots and other potential extraplexal axon donors were identified, distal targets were selected: 1) in the supraclavicular area; 2) by creating a new incision at the deltopectoral groove, splitting both muscles and sectioning the pectoral minor muscle to obtain access to the infraclavicular plexus; or 3) by creating a new incision, starting at the axilla and following the proximal medial brachial fossa. In the procedure described in the first technique, either no nerve graft or only a short one was generally required. Conversely, with techniques 2 and 3, a longer nerve graft was needed between the PN and the targets. A complete nerve section of the PN was used for the nerve transfer. Pertinent distal targets for elbow flexion recovery were, from proximal to distal, the anterior division of the superior trunk, the lateral cord, and the MCN or its branches for the biceps. Once donor and corresponding target nerves were selected, direct neurorrhaphy or autologous sural nerve grafts were used to bridge the gap. Nerve transfers were considered when the preoperative evaluation and intraoperative findings ruled out the presence of an available root that could serve as an alternative source of axons for grafting. Patients with partial root avulsion, documented on MRI, were excluded for PN transfer; and the same held for those patients in whom other nerve transfer options, such as the spinal accessory nerve to the MCN, were available. It is important to note 
that in all the cases included in this series, when the PN was transferred in an attempt to restore elbow flexion, this was the only donor to target elbow flexion.

Postoperative evaluations were performed on a regular basis (every 3 months) by at least two of the authors to reduce ascertainment bias. Potential side effects stemming from using the PN as an extraplexal donor for transfer were examined carefully. ${ }^{8}$ An intense rehabilitation program was started 3 weeks after surgery.

\section{Postoperative Clinical and Electrophysiological Evaluations}

The BMRC muscle-strength grading system-with ratings from 0 (no contraction) to 5 (normal strength) - was one of the methods used to assess the primary outcome of biceps flexion strength. Biceps strength, expressed in kilograms, also was determined by dynamometer (Baseline). This was accomplished by measuring the flexion force at the elbow in both arms, with the patient supine and the examiner positioned at his or her side holding the dynamometer with one or both hands. Each arm was tested three times, and the best score (maximum strength) recorded. Subsequently, a ratio was calculated by dividing the best score in the affected arm by the best score obtained in the healthy arm. This score would have a theoretical maximum value of 1.0 in the hypothetical case in which a patient exhibits complete recovery of elbow flexion strength on the affected side, and a minimum value of zero when no recovery is evident at all. No special attention was given to separately evaluating the biceps and brachialis muscles, as global functional flexion was measured.

Maximal elbow flexion strength was measured with the dynamometer during 1) a maximal inspiratory effort and 2) respiratory repose, after a maximal expiratory effort. In addition, the maximum degree of elbow flexion was obtained for each patient in the following situations: 1) after maximal inspiration, 2) during normal breathing, and 3) after maximal expiration.

Postoperative EMG testing of the biceps was performed at the final follow-up visit for each patient, using a concentric needle. Based on the data obtained from these studies, we developed a scale that allowed us to stratify measurements according to the number of motor unit potentials (MUPs) obtained in 150 milliseconds, ranging from a minimum score of 1 to a maximum score of 5 (Table 1). The number of MUPs was measured in three different situations: 1) during normal breathing with the arm at rest; 2) during sustained maximal inspiration with the arm at rest; and 3) during maximal voluntary biceps contraction, regardless of breathing status. Triceps EMG testing was also used as a control, employing the same protocol. Figure 1 summarizes the postoperative clinical and electrophysiological evaluations.

\section{Statistical Analysis}

All the outcomes of interest were continuous variables. They included both the measurements themselves, and differences in these measurements. Within-subject comparisons were conducted of the magnitude of these inspiration-to-expiration and normal-to-deep breathing
TABLE 1. Scale developed to quantify the biceps' response over 150 milliseconds, according to the number of MUPs recorded on EMG

\begin{tabular}{cc}
\hline Score on the Scale & No. of MUPs \\
\hline 1 & 0 \\
\hline 2 & $1-4$ \\
\hline 3 & $5-9$ \\
\hline 4 & $10-14$ \\
\hline 5 & $>15$ \\
\hline
\end{tabular}

differences using paired t-tests. Both the measurements themselves and breathing-related differences were also compared between patients with $<2.6$ years of postoperative follow-up (the median duration of follow-up in this series) and patients with $\geq 2.6$ years of follow-up, using Student t-tests for unpaired continuous variables and Pearson $\chi^{2}$ analysis for categorical variables such as patient sex and EMG MUP score.

Pearson correlation coefficients were calculated for each of the four paired outcomes: 1) biceps strength relative to the normal side during full inspiration and after full expiration; 2) the degrees of elbow flexion after full inspiration and after full expiration; 3) involuntary EMG activity during normal and deep breathing with the arm at rest; and 4) EMG activity during a maximal attempt of voluntary biceps contraction. The five outcomes of interest were: 1) inspiration-to-expiration differences in biceps strength relative to the normal side; 2 ) inspirationto-expiration differences in the maximal degrees of elbow flexion; 3) the degrees of involuntary elbow flexion during normal breathing; 4) the normal-to-deep breathing differences in involuntary EMG activity; and 5) EMG activity during maximum voluntary biceps muscle contraction. Pearson correlation coefficients were also calculated for the five outcomes of interest relative to patient age, time from injury to surgery, and duration of postoperative follow-up. For all correlation analyses, $\mathrm{r}$ values up to 0.30 were considered a weak correlation, from 0.31 to 0.70 a moderate correlation, and $\geq 0.71$ a strong correlation.

Since univariate analysis identified time to surgery and the inspiration-to-expiration difference in elbow flexion as statistically different between those with $<2.6$ versus $\geq$ 2.6 years of follow-up, simple linear regression analysis was performed, with inspiration-to-expiration difference in elbow flexion entered as the dependent variable, and both time to surgery (in months) and length of follow-up (in years) entered as independent variables, both by forward entry and by backward conditional entry.

All statistical tests, both univariate and multivariate, were 2-tailed, with $p=0.05$ set as the threshold for statistical significance, and $p$ values from 0.051 to 0.10 were considered borderline significant.

\section{Results}

Twenty-one patients fit the inclusion criteria and were deemed eligible for the study. Of these 21 patients, 19 (91\%) were male. Patient age ranged from 15 to 37 years, 


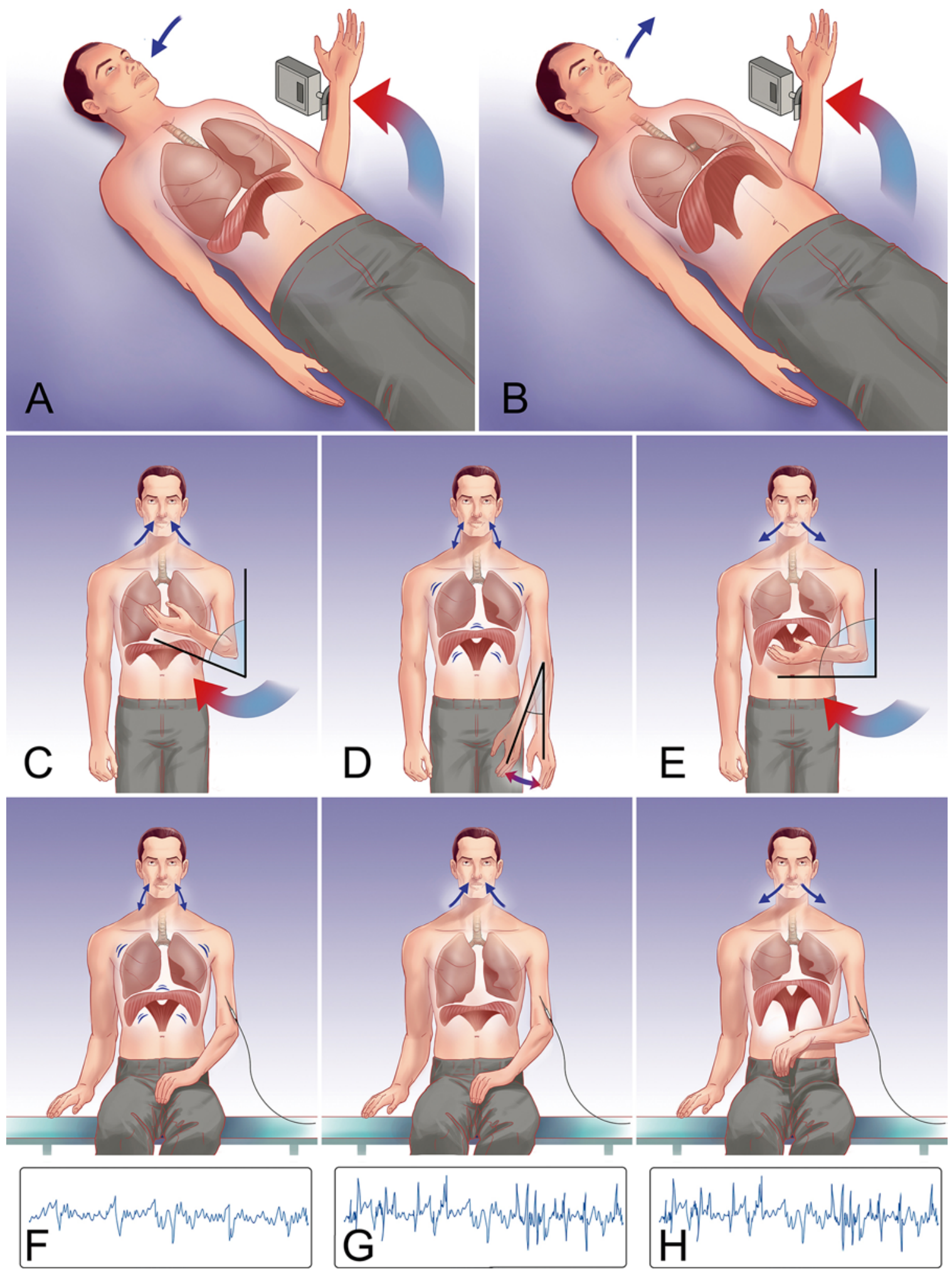

FIG. 1. Illustrations of the postoperative clinical and electrophysiological evaluations. A: Maximal strength on elbow flexion during maximal inspiration (the blue arrow shows air entering the lungs). B: The same evaluation after a maximal expiratory effort (the blue arrow shows the end of active expiration). C: The maximum degree of elbow flexion was measured after maximal inspiration (blue arrows show air entering the lungs). D: Degrees of spontaneous elbow movement while breathing normally (so-called "breathing arm"). E: Maximum degree of elbow flexion obtained after maximal expiration (blue arrows show air leaving the lungs). F: Postoperative EMG testing of the biceps was performed during normal breathing with the arm at rest (blue arrows show air entering and leaving the lungs). G: Same evaluation as panel $F$ during sustained maximal inspiration with the arm at rest. H: Same evaluation as shown in panel F, but during maximal voluntary biceps contraction, regardless of breathing status. Copyright Mariano Socolovsky. Published with permission. Figure is available in color online only. 
TABLE 2. Demographics, clinical characteristics, and surgical strategies used in patients of the sample

\begin{tabular}{|c|c|c|c|c|c|c|}
\hline $\begin{array}{l}\text { Pt } \\
\text { No. }\end{array}$ & $\begin{array}{l}\text { Age (yrs), } \\
\text { Sex }\end{array}$ & $\begin{array}{l}\text { Type of Lesion } \\
\text { (preop) }\end{array}$ & $\begin{array}{l}\text { Time From Trauma } \\
\text { to Op (mos) }\end{array}$ & $\begin{array}{c}\text { Time From Op to Data } \\
\text { Acquisition (yrs) }\end{array}$ & Surgical Techniques & $\begin{array}{l}\text { Graft Length }(\mathrm{cm}) \text { From PN } \\
\text { to Target for Elbow Flexion }\end{array}$ \\
\hline 1 & $19, \mathrm{M}$ & $\mathrm{C} 5-\mathrm{T} 1$ & 14 & 0.9 & PN-ADUT + XI-SP & 0 (no graft) \\
\hline 2 & $26, M$ & $\mathrm{C} 5-\mathrm{T} 1$ & 9.5 & 2.4 & PN-ADUT + XI-SP & 0 (no graft) \\
\hline 3 & $19, \mathrm{M}$ & $\mathrm{C} 5-\mathrm{T} 1$ & 9 & 1.1 & PN-MCN + XI-SP & 10 \\
\hline 4 & $18, \mathrm{M}$ & $\mathrm{C} 5-\mathrm{T} 1$ & 9 & 2 & PN-ADUT + XI-SP & 0 (no graft) \\
\hline 5 & $28, M$ & $\mathrm{C} 5-\mathrm{T} 1$ & 7 & 2.7 & PN-ADUT + XI-SP + C5-PDUT & 5 \\
\hline 6 & $32, \mathrm{M}$ & $\mathrm{C} 5-\mathrm{T} 1$ & 6 & 2.4 & PN-BBMC + XI-SP + C5-PDUT & 18 \\
\hline 7 & $35, \mathrm{M}$ & $\mathrm{C} 5-\mathrm{T} 1$ & 6 & 1.4 & PN-MCN + XI-SP & 10 \\
\hline 8 & $15, F$ & $\mathrm{C} 5-\mathrm{T} 1$ & 5 & 9.6 & PN-MCN + XI-SP & 18 \\
\hline 9 & $17, \mathrm{M}$ & C5-8 & 5 & 6.7 & PN-ADUT + XI-SP & 3 \\
\hline 10 & $18, \mathrm{M}$ & $\mathrm{C} 5-\mathrm{T} 1$ & 5 & 3.2 & PN-ADUT + XI-SP & 4 \\
\hline 11 & $31, \mathrm{M}$ & C5-T1 & 5 & 2.6 & PN-ADUT + XI-SP + C5-PDUT & 4 \\
\hline 12 & $21, \mathrm{M}$ & $\mathrm{C} 5-\mathrm{T} 1$ & 5 & 2.2 & PN-MCN + XI-SP & 11 \\
\hline 13 & $37, \mathrm{M}$ & $\mathrm{C} 5-\mathrm{T} 1$ & 4 & 2.8 & PN-ADUT + XI-SP & 4 \\
\hline 14 & $20, M$ & $\mathrm{C} 5-\mathrm{T} 1$ & 4 & 2.6 & PN-ADUT + XI-SP & 2 \\
\hline 15 & $23, \mathrm{M}$ & $\mathrm{C} 5-\mathrm{T} 1$ & 4 & 1.8 & PN-ADUT + XI-SP + C5-PDUT & 5 \\
\hline 16 & $18, \mathrm{M}$ & $\mathrm{C} 5-\mathrm{T} 1$ & 4 & 0.8 & PN-ADUT + XI-SP & 0 (no graft) \\
\hline 17 & $27, \mathrm{M}$ & $\mathrm{C} 5-\mathrm{T} 1$ & 3 & 8.8 & PN-BBMC + XI-SP & 22 \\
\hline 18 & $28, \mathrm{M}$ & $\mathrm{C} 5-\mathrm{T} 1$ & 3 & 6.9 & PN-MCN + XI-SP & 18 \\
\hline 19 & $24, F$ & C5-8 & 3 & 5.7 & PN-ADUT + XI-SP + C5-PDUT & 4 \\
\hline 20 & $24, \mathrm{M}$ & $\mathrm{C} 5-\mathrm{T} 1$ & 3 & 2.9 & PN-ADUT + XI-SP + C5-PDUT & 3 \\
\hline 21 & $31, \mathrm{M}$ & $\mathrm{C} 5-\mathrm{T} 1$ & 2 & 5.2 & $\mathrm{PN}-\mathrm{MC}+\mathrm{XI}-\mathrm{SP}$ & 18 \\
\hline
\end{tabular}

$\mathrm{ADUT}=$ anterior division of the upper trunk; BBMC = biceps branch of the musculocutaneous nerve; $\mathrm{PDUT}=$ posterior division of the upper trunk; $\mathrm{Pt}=\mathrm{patient} ; \mathrm{XI}-\mathrm{SP}=$ spinal accessory to suprascapular nerve transfer.

with a mean of 24.3 years and a median of 24 years. The mean interval from trauma to surgery was 5.5 months, ranging from 2 to 12 months, and a median of 5 months. The mean duration of follow-up was 2.6 years, ranging from 10 months to 9.6 years. In accordance with our previous report, ${ }^{8}$ no patient experienced any clinical or functional respiratory complications after PN sectioning across the entire series. Tables 2 and 3 summarize patient characteristics.

Across the entire sample $(n=21)$, mean biceps strength was 0.21 after maximal expiration versus 0.29 upon maximal inspiration, a difference of $0.08(\mathrm{t}=4.97, \mathrm{p}<0.001)$. Similarly, there was almost a $21^{\circ}$ difference in maximum elbow flexion, from $88.8^{\circ}$ after expiration to $109.5^{\circ}$ during maximal inspiration $(\mathrm{t}=5.05, \mathrm{p}<0.001)$. These two sets of paired variables were both strongly, directly correlated $(\mathrm{r}=0.85, \mathrm{p}<0.001$, and $\mathrm{r}=0.78, \mathrm{p}<0.001$, respectively; Table 4). Involuntary elbow flexion during normal breathing (the so-called breathing arm) was present in 18 of the 21 subjects $(86 \%)$, ranging from $10^{\circ}$ to $50^{\circ}$ in the 18 with some involuntary respiration-linked movement, and averaging almost $20^{\circ}$ across the entire sample.

With respect to EMG activity, measuring involuntary activity with the biceps muscle at rest and EMG activity during biceps muscle contraction, there were statistically significant direct correlations between readings taken during normal breathing and during deep breathing, which were moderate $(r=0.66, p<0.001)$ and extremely strong $(r$
$=0.94, \mathrm{p}<0.001)$, respectively. Also, involuntary activity differed significantly between normal and deep breathing (2.14 vs $3.14, \mathrm{t}=4.58, \mathrm{p}<0.001$; Table 4$)$.

To study the way our results changed with different follow-up times, we established the mean duration of follow-

TABLE 3. Mean values obtained from the sample

\begin{tabular}{lcc}
\hline \multicolumn{1}{c}{ Variable } & Value & Range \\
\hline$\%$ males & 90.5 & $\mathrm{NA}$ \\
\hline Mean age (yrs) & 24.3 & $15-37$ \\
\hline Median age (yrs) & 24 & $15-37$ \\
\hline Mean time from trauma to op (mos) & 5.5 & $2-12$ \\
\hline Mean (median) duration of follow-up (yrs) & $2.6(2.6)$ & $0.8-9.6$ \\
\hline Mean biceps strength after expiration & 0.21 & $0.04-0.50$ \\
\hline Mean biceps strength on maximal inspiration & 0.29 & $0.10-0.43$ \\
\hline Mean difference & 0.08 & -0.10 to 0.21 \\
\hline Mean elbow flexion after expiration $\left(^{\circ}\right)$ & 88.8 & $10-150$ \\
\hline $\begin{array}{l}\text { Mean elbow flexion during maximal inspira- } \\
\text { tion }\left(^{\circ}\right)\end{array}$ & 109.5 & $50-180$ \\
\hline Mean difference $\left(^{\circ}\right)$ & 20.7 & $0-65$ \\
\hline Spontaneous arm movement while breath- & 19.8 & $0-75$ \\
ing $\left({ }^{\circ}\right)$ & & \\
\hline
\end{tabular}

NA = not applicable. 
TABLE 4. Analysis of paired outcomes

\begin{tabular}{lccccccc}
\hline & & & \multicolumn{2}{c}{ Breathing } & & & \\
\multicolumn{1}{c}{ Outcome } & Expiration & Inspiration & Normal & Deep & Difference & Correlation \\
\hline Biceps strength & 0.21 & 0.29 & & & $t=4.97, p<0.001$ & $r=0.85, p<0.001$ \\
\hline Elbow flexion $\left(^{\circ}\right)$ & 88.8 & 109.5 & & & $t=5.05, p<0.001$ & $r=0.78, p<0.001$ \\
\hline Involuntary muscle activity on EMG & & & 2.14 & 3.14 & $t=4.58, p<0.001$ & $r=0.66, p<0.001$ \\
\hline
\end{tabular}

up (2.6 years) as a landmark to divide our patients into two groups. Table 5 compares patients with durations of follow-up above or equal to, versus below, the mean duration of follow-up. Four statistically significant differences between these two patient groups were detected: the time from trauma to surgery $(\mathrm{p}=0.017)$; mean length of follow-up, which differed by a mean 3.3 years $(\mathrm{p}=0.001)$; in terms of outcomes, the mean difference in degrees of elbow flexion obtained between full expiration and maximal inspiration ( $\mathrm{p}=0.027)$; and, as an ordinal variable, the EMG MUP score for the biceps $\left(\chi^{2}=9.33\right.$, df $=3, \mathrm{p}$ $=0.025)$. Figure 2 graphically demonstrates the lack of any significant difference in these two patient groups pertaining to inspiration-to-expiration differences in biceps strength, and in both EMG evaluations comparing normal and deep breathing. Conversely, Fig. 3 clearly demonstrates a marked difference in maximal elbow flexion degrees between inspiration and expiration, though there is no meaningful difference in spontaneous elbow flexion with breathing.

Because the variable "time from trauma to surgery" might have confounded our analysis of the association between the duration of follow-up and breathing's effect on elbow flexion, we entered the two variables "time to sur-

TABLE 5. Comparing patients with $<2.6$ versus $\geq 2.6$ years (median) follow-up

\begin{tabular}{|c|c|c|c|c|}
\hline \multirow[b]{2}{*}{ Variable } & \multicolumn{2}{|c|}{ Yrs of Follow-Up } & \multirow[b]{2}{*}{ t Score $/ \chi^{2}$} & \multirow[b]{2}{*}{ p Value } \\
\hline & $<2.6$ & $\geq 2.6$ & & \\
\hline No. of pts & 9 & 12 & & \\
\hline Mean age (yrs) & 23.4 & 25.0 & $t=0.55$ & 0.59 \\
\hline Mean time between injury \& op (mos) & 7.4 & 4.1 & $t=2.84$ & 0.017 \\
\hline Mean length of follow-up (yrs) & 1.7 & 5.0 & $t=4.31$ & 0.001 \\
\hline Mean biceps strength on expiration & 0.17 & 0.21 & $t=0.72$ & 0.47 \\
\hline Mean biceps strength on inspiration & 0.26 & 0.30 & $t=0.83$ & 0.42 \\
\hline Mean difference & 0.09 & 0.09 & $t=0.06$ & 0.95 \\
\hline Mean elbow flexion on expiration $\left({ }^{\circ}\right)^{*}$ & 80.1 & 95.0 & $t=0.98$ & 0.35 \\
\hline Mean elbow flexion on inspiration $\left({ }^{\circ}\right)^{*}$ & 112.8 & 107.1 & $t=0.51$ & 0.51 \\
\hline Mean difference $\left({ }^{\circ}\right)^{*}$ & 32.2 & 12.1 & $t=2.56$ & 0.027 \\
\hline Spontaneous arm movement while breathing & 20.0 & 17.9 & $t=0.25$ & 0.81 \\
\hline \multicolumn{5}{|l|}{ EMG findings for biceps } \\
\hline Involuntary activity during normal breathing & 2.3 & 2.0 & $t=0.44$ & 0.66 \\
\hline Involuntary activity during deep breathing & 3.1 & 3.0 & $t=0.22$ & 0.83 \\
\hline Difference between normal \& deep breathing & 0.9 & 1.0 & $t=0.27$ & 0.79 \\
\hline Muscle activity during muscle contraction & 4.1 & 4.7 & $t=1.37$ & 0.19 \\
\hline$\%$ achieving MUP score = 1 & 0.0 & 0.0 & & \\
\hline$\%$ achieving MUP score $=2$ & 11.1 & 0.0 & & \\
\hline$\%$ achieving MUP score $=3$ & 0.0 & 16.7 & $\chi^{2}=9.33$ & 0.025 \\
\hline$\%$ achieving MUP score $=4$ & 44.4 & 0.0 & & \\
\hline$\%$ achieving MUP score $=5$ & 44.4 & 83.3 & & \\
\hline \multicolumn{5}{|l|}{ EMG findings for biceps relative to triceps } \\
\hline Involuntary activity during normal breathing & 1.3 & 1.0 & $t=0.44$ & 0.66 \\
\hline Involuntary activity during deep breathing & 2.1 & 2.0 & $t=0.22$ & 0.83 \\
\hline Difference between normal \& deep breathing & 0.9 & 1.0 & $t=0.27$ & 0.79 \\
\hline Muscle activity during muscle contraction & 3.1 & 3.5 & $t=0.88$ & 0.39 \\
\hline
\end{tabular}

Boldface type indicates statistical significance.

* This analysis excludes the 1 patient (case 4 ) whose elbow flexion increased by $55^{\circ}$ on inspiration. 


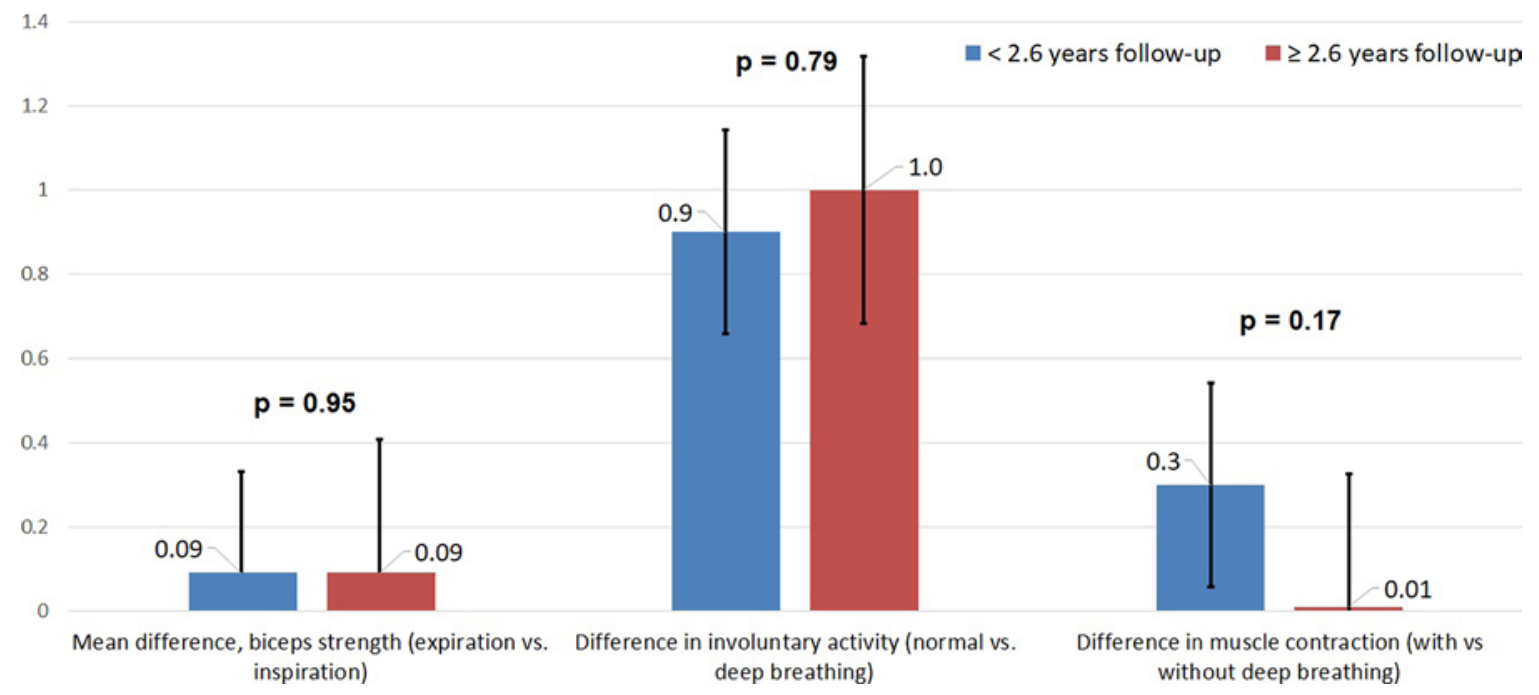

FIG. 2. Graph of respiration-related differences in muscle strength and EMG activity. Figure is available in color online only.

gery" and "duration of follow-up" into a simple linear regression model to determine which of these two factors, if either, was the better predictor of the inspiration-to-expiration difference in elbow flexion range of motion (ROM). In the final model, the length of postoperative follow-up was retained as a borderline predictor $(\mathrm{p}=0.09)$, while time to surgery was excluded $(\mathrm{p}=0.63)$.

Table 6 provides Pearson correlation coefficients ( $\mathrm{r}$ values) and their level of statistical significance ( $\mathrm{p}$ values) between three continuous-variable patient characteristics (age, time to surgery, and duration of postoperative followup) and our five main outcomes of interest, all assessing the impact of breathing on elbow function or physiology. Among the 15 correlation coefficients calculated $(3 \times 5)$, only one was statistically different than zero, which was the correlation between the duration of postoperative follow-up and the difference in degrees of elbow flexion between maximum inspiration and expiration. This correlation $(\mathrm{r}=-0.38)$ was inverse in direction (meaning that increased follow-up was associated with a reduced effect of breathing) and moderate in magnitude. Time to surgery and the duration of follow-up also were moderately, inversely correlated $(\mathrm{r}=-0.47, \mathrm{p}=0.03)$. The difference in the biceps MUP score between an inactive biceps during

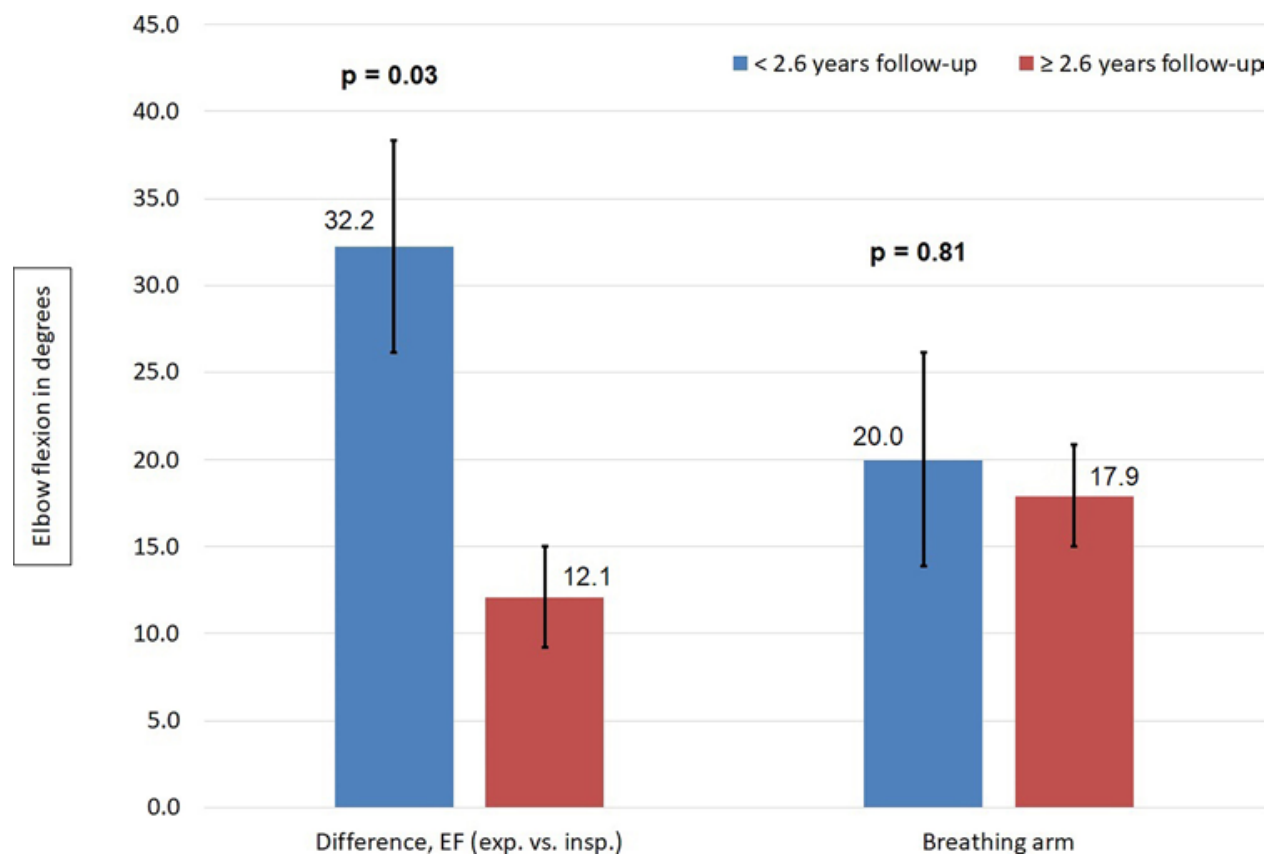

FIG. 3. Respiration-related differences in elbow flexion (EF), measured in degrees. exp. $=$ expiration; insp. $=$ inspiration. Figure is available in color online only. 
TABLE 6. Pearson correlation coefficients and their significance for the different outcomes of interest

\begin{tabular}{|c|c|c|c|}
\hline \multirow[b]{2}{*}{ Outcome of Interest } & \multicolumn{3}{|c|}{ Baseline Characteristic } \\
\hline & Pt Age & Time to Op & Length of Follow-Up \\
\hline Difference in biceps strength (inspiration vs expiration) & $0.19(0.41)$ & $0.20(0.37)$ & $-0.13(0.33)$ \\
\hline Difference in maximal elbow flexion degrees (inspiration vs expiration) & $-0.14(0.54)$ & $0.09(0.71)$ & $-0.38(0.08)$ \\
\hline Degrees of involuntary elbow flexion during breathing (breathing arm) & $0.06(0.81)$ & $0.13(0.58)$ & $-0.29(0.21)$ \\
\hline Difference in involuntary activity (normal vs deep breathing) & $0.04(0.87)$ & $0.14(0.53)$ & $-0.09(0.63)$ \\
\hline MUP score & $0.006(0.98)$ & $0.21(0.36)$ & $-0.08(0.73)$ \\
\hline
\end{tabular}

Data given as Pearson correlation coefficients ( $p$ values). Note that time to surgery and duration of follow-up were moderately, inversely correlated: $r=-0.47, p=0.03$.

normal and deep breathing was not correlated with the final biceps MUP score $(\mathrm{r}=-0.19, \mathrm{p}=0.40)$.

\section{Discussion}

At final follow-up in our cohort of 21 patients with TBPI who underwent a PN transfer, there were statistically significant differences between measurements made after full inspiration and full expiration. These differences included: 1) a mean 0.08 greater difference between the healthy versus affected arm during full inspiration than after full expiration in biceps strength; 2) a mean $21^{\circ}$ greater difference between the healthy versus affected arm in elbow flexion, also in full inspiration over full expiration; and 3) in EMG readings for both voluntary and involuntary movements.

Interestingly, a highly significant difference was apparent for respiration-related EMG activity with the biceps at rest; however, during biceps contraction, no respiration-related EMG effect was noted. This latter finding is probably related to the elevated number of MUPs that appeared during biceps contraction, which likely hid any influence of breathing. The numbers of MUPs, on an ordinal scale, during biceps contraction for normal and deep breathing were very strongly correlated, reflecting the original function of the PN to innervate the hemidiaphragm. All these findings might demonstrate that, in our cohort of patients after PNMCN transfer, the influence of inspiration (and therefore, PN firings) on elbow flexion was considerable. Because our scale has not yet been validated, however, it should be used with caution, i.e., as a surrogate marker for muscle activation when comparing maximal inspiration versus expiration data, but not as a robust outcome measure.

Also notable is that we were able to statistically demonstrate that this influence of breathing on elbow flexion decreased over time, but only with respect to ROM, and not biceps strength or EMG activity. The difference between expiration and inspiration in the degrees of maximal elbow flexion was significantly larger among those with 2.6 or fewer years of follow-up than in those with longer follow-up. Brain plasticity occurring after PN-MCN transfer might be linked to these changes. Conversely, the difference in biceps strength between inspiration and expiration tended to be more stable over time in our series. No statistically significant influence of follow-up duration was apparent for the effect of breathing over time on biceps strength, involuntary EMG muscle activity during biceps rest, or voluntary EMG muscle activity during biceps con- traction. None of these three variables was even weakly correlated with the length of follow-up. Even though we could theorize that, perhaps, some effect of time might become apparent in a larger patient population, the very low Pearson correlation coefficients that our sample generated argue against even a statistically significant effect (if detected through sheer sample size) having any clinical relevance. Further investigation is needed to clarify the reason behind this lack of any effect of time on these measurements, given the clear correlation between follow-up time and elbow ROM.

The concept of a breathing arm (one that moves involuntarily during respiration) was first proposed by Swift in $1994 .^{10}$ We have identified only two other papers describing patients with a breathing arm in the literature: one a study with 26 patients, ${ }^{5}$ and the other a solitary case report about a 9-year-old boy. ${ }^{2}$ Originally, the term "breathing arm" described a phenomenon that occurs after a traction injury involving the brachial plexus that is treated conservatively. After the injury, if spontaneously regenerating axons that stem from $\mathrm{C} 4$ and originally run to the PN misroute and enter $\mathrm{C} 5$, those axons can reach the MCN via the anterior division of the superior trunk. If a needle is inserted into the involved biceps at rest, breathing-related EMG activity will be observed in the biceps. Later, the term "breathing arm" was expanded to incorporate the movement that can occur from muscles that have been reinnervated by respiratory nerves, whether the ICN or the PN.

We observed breathing arms in 18 of our 21 patients, with an average of roughly $20^{\circ}$ of involuntary elbow flexion detected across our entire sample. Although those with less than the median duration of follow-up averaged $2.1^{\circ}$ more involuntary flexion than their counterparts, this difference was far from statistically significant. In contrast, on correlation analysis, this measurement was the second most strongly correlated with the duration of follow-up $(\mathrm{r}$ $=-0.29, \mathrm{p}=0.21$ ), suggesting that at least a tendency toward a weak inverse correlation might have been detected if our patient sample had been larger. Therefore, similarly to what happens for maximal degrees of elbow flexion, as time passes the influence of inspiration on the so-called breathing arm could tend to decrease over time, contrary to what is observed for biceps strength and EMG activity.

According to some reports in the literature, PN-MCN transfers might generate better and more predictable results than ICN-MCN nerve transfers. ${ }^{8}$ The larger number of available motor donors might explain these results. Re- 
garding the strong influence that inspiratory movements appear to have on elbow flexion observed in our and other series, this could be the cost for the enhanced results of PN to $\mathrm{MCN}$ relative to $\mathrm{ICN}$ to $\mathrm{MCN}$ transfers. However, any discussion about the potential eventual risks of long-term adverse respiratory effects after a $\mathrm{PN}$ to $\mathrm{MCN}$ transfer, risks for which complete consensus does not yet exist, is beyond the scope of our present study.

The PNs and ICNs have somewhat different functions during respiration, which might help to explain some of the findings in the current study. One major difference is that the PN primarily fires during inspiration, ${ }^{6}$ while rib-cage muscles (innervated by, among others, ICNs) are required only during expiration, and only in certain circumstances, because normal expiration is usually a passive motion. Scenarios in which active expiration is required include engaging in heavy exercise and singing, when breathing must either be more rapid or more controlled than at rest, respectively. Interpreting the results of the current study, we can theorize that PN-MCN transfers are, therefore, less disconnected from breathing than ICN-MCN transfers, as suggested previously.

The ICN and PN have different networks of interneurons within the cortical and subcortical zones of the primary motor cortex. In fact, some authors focus on this difference and promote it using a differentiated physiotherapy program for patients who undergo a PN transfer for biceps repair and an ICN transfer for triceps repair, instructing patients to perform their inspiration- and expiration-linked exercises, thereby working elbow flexion and extension separately. ${ }^{3}$

Some investigators have argued against reinnervating two antagonistic muscles (such as the biceps and triceps) using both the PNs and ICNs, as brain plasticity has limits. ${ }^{11}$ When healthy individuals lift a heavy object, for example, the ICN fires in synchrony with the MCN to stabilize the rib cage. Conversely, the PN, which is more devoted to inspiration during diaphragm contraction, could have different pathways and, therefore, might be slower and less able to attain complete independence of movements from respiration. Yet, it has also been observed that lifting a heavy weight with both arms in flexion many times is associated with full inspiration, an observation likely related to there being a common pathway for elbow flexion and inspiration mediated by interneurons and previously existing intercortical connections. As with ICN-MCN transfers, brain plasticity might be involved in the mediation of the cortical changes related to both movements. A study comparing ICN-MCN and PN-MCN transfers, in terms of the degree of independence achieved for elbow flexion, is clearly necessary to clarify this point.

From a clinical point of view, it is important to state that patients undergoing $\mathrm{PN}$ to MCN transfers do not complain of uncomfortable movements of the arm while breathing, despite the theoretical possibility that spontaneous flexion of the biceps might be considered annoying and/or inconvenient. Perhaps the gradual waning of this effect over time, as our data suggest, explains this acceptance. Interestingly, these patients do tend to adopt simultaneous inspiration with biceps flexion when they want to lift heavy objects, potentially to enhance their strength (which was, albeit nonsignificantly, $10 \%$ greater than after expiration in our study). Then, once the heavy object is lifted, they resume normal breathing. It is possible that their combining a deep breath with lifting is nothing more than what healthy individuals tend to do. On the other hand, it also could be a learned response. We are currently examining this issue in our own patients.

One major strength of the current study is that it is the first study to assess for any link between respiration and biceps function following PN-MCN transfers. Another strength is the homogeneity of our patient sample, with all patients restricted in age between mid-adolescence and relatively young adulthood, having similar injuries, and undergoing the same essential procedure.

The major limitation of our study pertains to the relatively small sample size. However, despite just having 21 patients, we still were able to document a clinically and statistically significant waning of breathing-related effects over time on the maximal degrees of elbow flexion. Perhaps with a larger sample we could have detected similar, statistically significant correlations between the duration of postoperative follow-up and our other outcomes of interest, like the strength of involuntary elbow flexion observed while breathing. On the other hand, in our sample, the magnitudes of all these other correlations were very weak, such that variance in the duration of follow-up ranged from explaining just $0.8 \%$ to no more than $8.4 \%$ of the variance in these other outcomes.

\section{Conclusions}

Our results clearly suggest that the impact of breathing on elbow function is considerable when patients undergo a PN transfer to reconstruct elbow function after a BPI, both clinically and electromyographically. However, there appears to be some waning of this influence over time, a process that might involve brain plasticity. This waning effect could impact elbow range of motion more than biceps muscle strength and EMG activity. It would be reasonable to inform patients about the breathing-biceps function link prior to performing PN transfers. Further research is necessary to corroborate all these conjectures.

\section{References}

1. Anastakis DJ, Malessy MJ, Chen R, Davis KD, Mikulis D: Cortical plasticity following nerve transfer in the upper extremity. Hand Clin 24:425-444, vi-vii, 2008

2. Carlstedt T, Anand P, Htut M, Misra P, Svensson M: Restoration of hand function and so called "breathing arm" after intraspinal repair of C5-T1 brachial plexus avulsion injury. Case report. Neurosurg Focus 16(5):E7, 2004

3. Dahlin LB, Andersson G, Backman C, Svensson H, Björkman A: Rehabilitation, using guided cerebral plasticity, of a brachial plexus injury treated with intercostal and phrenic nerve transfers. Front Neurol 8:72, 2017

4. Fraiman D, Miranda MF, Erthal F, Buur PF, Elschot M, Souza L, et al: Reduced functional connectivity within the primary motor cortex of patients with brachial plexus injury. Neuroimage Clin 12:277-284, 2016

5. Htut M, Misra VP, Anand P, Birch R, Carlstedt T: Motor recovery and the breathing arm after brachial plexus surgical repairs, including re-implantation of avulsed spinal roots into the spinal cord. J Hand Surg Eur Vol 32:170-178, 2007 
6. Lumb AB: Nunn's Applied Respiratory Physiology, ed 8. Amsterdam: Elsevier, 2016, pp 73-89

7. Malessy MJ, van Dijk JG, Thomeer RT: Respiration-related activity in the biceps brachii muscle after intercostal-musculocutaneous nerve transfer. Clin Neurol Neurosurg 95 (Suppl):S95-S102, 1993

8. Socolovsky M, di Masi G, Bonilla G, Domínguez Paez M, Robla J, Calvache Cabrera C: The phrenic nerve as a donor for brachial plexus injuries: is it safe and effective? Case series and literature analysis. Acta Neurochir (Wien) 157:1077-1086, 2015

9. Socolovsky M, Malessy M, Lopez D, Guedes F, Flores L: Current concepts in plasticity and nerve transfers: relationship between surgical techniques and outcomes. Neurosurg Focus 42(3):E13, 2017

10. Swift TR: The breathing arm. Muscle Nerve 17:125-129, 1994

11. Zheng MX, Xu WD, Qiu YQ, Xu JG, Gu YD: Phrenic nerve transfer for elbow flexion and intercostal nerve transfer for elbow extension. J Hand Surg Am 35:1304-1309, 2010

\section{Disclosures}

The authors report no conflict of interest concerning the materials or methods used in this study or the findings specified in this paper.

\section{Author Contributions}

Conception and design: Socolovsky, Bonilla, Di Masi, Conti. Acquisition of data: Socolovsky, Bonilla, Di Masi, Conti, Lovaglio. Analysis and interpretation of data: Socolovsky, Bonilla, Di Masi, Conti, Lovaglio. Drafting the article: Socolovsky, Lovaglio. Critically revising the article: Malessy, Conti, Lovaglio. Reviewed submitted version of manuscript: Socolovsky, Malessy, Bonilla, Di Masi. Approved the final version of the manuscript on behalf of all authors: Socolovsky. Statistical analysis: Socolovsky, Malessy. Administrative/technical/material support: Socolovsky. Study supervision: Socolovsky.

\section{Correspondence}

Mariano Socolovsky: University of Buenos Aires School of Medicine, Buenos Aires, Argentina. marianosocolovsky@gmail.com. 